\title{
Peroxisomal Dysfunction and Oxidative Stress in Neurodegenerative Disease: A Bidirectional Crosstalk
}

\author{
Marc Fransen, Iulia Revenco, Hongli Li, Cláudio F Costa, \\ Celien Lismont, and Paul P Van Veldhoven
}

\author{
Laboratory of Lipid Biochemistry and Protein Interactions, Department of Cellular and \\ Molecular Medicine, KU Leuven, Belgium \\ Corresponding author: Marc Fransen (marc.fransen@kuleuven.be)
}

\begin{abstract}
Peroxisomes are multifunctional organelles best known for their role in cellular lipid and hydrogen peroxide metabolism. In this chapter, we review and discuss the diverse functions of this organelle in brain physiology and neurodegeneration, with a particular focus on oxidative stress. We first briefly summarize what is known about the various nexuses between peroxisomes, the central nervous system, oxidative stress, and neurodegenerative disease. Next, we provide a comprehensive overview of the complex interplay between peroxisomes, oxidative stress, and neurodegeneration in patients suffering from primary peroxisomal disorders. Particular examples that are discussed include the prototypic Zellweger spectrum disorders and $\mathrm{X}$-linked adrenoleukodystrophy, the most prevalent peroxisomal disorder. Thereafter, we elaborate on secondary peroxisome dysfunction in more common neurodegenerative disorders, including Alzheimer's disease, Parkinson's disease, and multiple sclerosis. Finally, we highlight some issues and challenges that need to be addressed to progress towards therapies and prevention strategies preserving, normalizing, or improving peroxisome activity in patients suffering from neurodegenerative conditions.
\end{abstract}

Keywords: Peroxisome; Redox balance; Peroxisomal disorder; Mitochondria; Central nervous system; Neurological disease 


$\begin{array}{ll}\text { Abbreviations } \\ \text { AD } & \text { Alzheimer's disease } \\ \text { ABCD } & \text { ATP-binding cassette subfamily D } \\ \text { ALDP } & \text { Adrenoleukodystrophy protein } \\ \text { ALS } & \text { Amyotrophic lateral sclerosis } \\ \text { CNS } & \text { Central nervous system } \\ \text { DAO } & \text { D-amino acid oxidase } \\ \text { DHA } & \text { Docosahexaenoic acid } \\ \text { EAE } & \text { Experimental allergic encephalomyelitis } \\ \text { MS } & \text { Multiple sclerosis } \\ \text { PD } & \text { Parkinson's disease } \\ \text { PEX } & \text { Peroxin } \\ \text { PPA } & \text { Peroxisome proliferating agent } \\ \text { PPAR } & \text { Peroxisome proliferator activated receptor } \\ \text { PUFA } & \text { Polyunsaturated fatty acid } \\ \text { ROS } & \text { Reactive oxygen species } \\ \text { VLCFA } & \text { Very-long-chain fatty acid } \\ \text { X-ALD } & \text { X-linked adrenoleukodystrophy } \\ \text { ZSD } & \text { Zellweger spectrum disorder }\end{array}$

\section{Peroxisomes and the Central Nervous System}

Peroxisomes are morphologically and functionally remarkably plastic organelles that play a prominent role in diverse metabolic and signaling pathways [22]. In humans, they are pivotal for the oxidative degradation of a broad range of fatty acids and D-amino acids as well as the biosynthesis of plasmalogens, bile acids, and all-cis4,7,10,13,16,19-docosahexaenoic acid (DHA; 22:6 n-3) [22, 51]. Peroxisomes can also serve as a source or sink for hydrogen peroxide $\left(\mathrm{H}_{2} \mathrm{O}_{2}\right)$ [29]. Given that (i) this non-radical reactive oxygen species (ROS) acts as a physiologically important redox signaling messenger, (ii) plasmalogens and DHA function as potential reservoirs for lipid second messengers (e.g., prostaglandins, leukotrienes, thromboxanes, resolvins, and neuroprotectins), and (iii) some of these bioactive lipids can be degraded through peroxisomal $\beta$-oxidation, peroxisomes have the intrinsic capability to mediate and modulate redox- and lipid-driven signaling cascades [28]. Besides this, they 
have emerged as indispensable signaling platforms in innate immunity and inflammation [13]. To perform their functions effectively, peroxisomes must communicate and cooperate with other subcellular compartments, including mitochondria [16], and defects in one or more of these functions often result in a range of neurological symptoms and brain pathology (see Section 4).

Peroxisomes are present in all major cell types of the central nervous system (CNS), including neurons, astrocytes, oligodendrocytes, microglia, and endothelial cells [42], their abundance and intracellular distribution can differ between cell types. For example, peroxisomes in white matter tracts are highly abundant in myelinforming oligodendrocytes, but only sparsely present in axonal projections [24]. In comparison to other tissues, brain peroxisomes are smaller in size [2]. In addition, their abundance varies between different brain areas and significantly decreases during postnatal development [42]. From the functional point of view, peroxisomes play diverse roles in brain physiology: they (i) provide key metabolites (e.g., DHA and plasmalogens) for the formation and maintenance of myelin membranes and neuronal signaling, (ii) degrade important mediators of lipotoxicity (e.g., hydroperoxy fatty acids, very-longchain fatty acids (VLCFAs), and the dietary fatty acid phytanic acid and its metabolite pristanic acid), (iii) metabolize D-serine and Daspartate, two endogenous molecules involved in brain neurotransmission, synaptic plasticity, and neurodegeneration, and (iv) play a role in the maintenance of redox balance ([2]; and references therein) (Fig. 1). Finally, it is interesting to note that, despite the fact that oligodendrocyte-, astrocyte- as well as neuron-selective inactivation of peroxisomes in mice adversely affects the animals' postnatal thriving, only the oligodendrocyte-specific inactivation results in a reduced lifespan [52].

\section{Neurodegenerative Disease and Oxidative Stress}

The CNS, which comprises the brain and spinal cord, is made up of complex networks of functionally diverse cell types that control virtually all activities of the human body and mind. The brain is intrinsically vulnerable to oxidative stress, a phenomenon that can be 
explained by - among others - its high oxygen consumption, rich content of peroxidation-sensitive polyunsaturated fatty acids (PUFAs), regionally high abundance of redox-active transition metals, and relatively modest antioxidant defenses [9]. Increased oxidative stress is one of the key hallmarks of neurodegenerative disorders, the most common being Alzheimer' disease, Parkinson's disease, and multiple sclerosis. Other distinctive features include mitochondrial dysfunction, protein misfolding and aberrant localization, inflammation, and neuronal cell death [8]. Importantly, despite the fact that oxidative stress has been associated with the onset and progression of neuropathological alterations, it is not yet clear whether disturbances in the cellular redox balance represent a secondary response to brain damage or play a causative role in disease development.

\section{Peroxisomes and Cellular Redox Balance}

Mammalian peroxisomes contain about 100 different proteins, of which approximately a dozen generate $\mathrm{H}_{2} \mathrm{O}_{2}$ as metabolic byproduct [41]. In addition, one of the most abundant peroxisomal proteins is catalase, an enzyme that efficiently decomposes $\mathrm{H}_{2} \mathrm{O}_{2}$ to $\mathrm{H}_{2} \mathrm{O}$ and $\mathrm{O}_{2}$ without consuming reducing equivalents. Currently, there is compelling empirical evidence that peroxisomes serve a key role in cellular redox balance and stress responses ([29]; and references therein). In the context of this chapter, it needs to be emphasized that disturbances in peroxisomal (redox) metabolism can rapidly perturb mitochondrial redox state and function, a key feature of neurodegenerative diseases. Unfortunately, little is known about how disturbances in peroxisomal redox metabolism affect other subcellular compartments. Given that the main focus of this thematic review lies on the interplay between peroxisome (dys)function and oxidative stress in neurodegenerative disease (see Sections 4 and 5), we refer the reader to other recent reviews for detailed discussions on how peroxisomes may act as redox signaling hubs [29] and why these organelles need to maintain a healthy relationship with mitochondria [16]. 


\section{Peroxisomal Dysfunction, Oxidative Stress, and Neurodegeneration}

The observation that virtually all patients with peroxisomal dysfunction present with neurological symptoms clearly highlights that maintenance of a healthy peroxisomal population is critical for neuronal health [2]. Depending on the metabolic pathways and cell types involved, the CNS pathology may be more or less severe [54]. Symptoms can range from abnormalities in neuronal migration or differentiation to defects in the formation or maintenance of central white matter and even post-developmental neuronal degeneration [2]. The exact immuno- and inflammatory dysregulations causing these injuries remain unclear, but potential factors may include reduced plasmalogen content [14], toxic accumulation of peroxisomal $\alpha$ - (e.g., phytanic acid) and $\beta$-oxidation (e.g., pristanic acid and VLCFAs) substrates, and changes in peroxisomal $\mathrm{H}_{2} \mathrm{O}_{2}$ metabolism and signaling (Fig. 2).

Plasmalogens have been shown to protect myelin from oxidative damage [14], and a general shortage of this class of glycerophospholipids in mitochondrial membranes has been reported to reduce respiratory chain activity and ATP production, a condition negatively affecting cerebral energy metabolism [6]. Also phytanic and pristanic acid as well as the VLCFAs docosanoic acid (C22:0), tetracosanoic acid (C24:0), and hexacosanoic acid (C26:0) have been reported to exert harmful effects on neural cells and brain tissues through mitochondrial dysfunction and oxidative stress, at least in vitro. Indeed, in vitro studies with neural cells have shown that low micromolar concentrations of these lipids can distort mitochondrial respiration, $\mathrm{Ca}^{2+}$ retention, and ROS production, thereby impairing cell physiology and viability ([43, 60]; and references therein); and ex-vivo studies on brain cortex and cerebellum of young rats have shown that micromolar concentrations of both phytanic and pristanic acid elicit lipid and protein oxidative damage $[7,26]$. However, in vivo injection of phytanic acid (estimated final concentration: $450-500 \mu \mathrm{M}$ ) into the cerebellum of adolescent rats did only provoke oxidative damage to lipids [3]. Interestingly, treatment of primary mixed neural cultures of rat hippocampus revealed that the pathological consequences of VLCFA accumulation are more severe for myelin- 
producing oligodendrocytes than for astrocytes and neurons [43]. In addition, the disease pathology associated with the accumulation of VLCFAs in the CNS appears to be more severe in humans than in mice ([11]; and references therein).

A major concern regarding the studies referred to in the previous paragraph is that the administered concentrations of fatty acids are probably way outside the physiological range. In this context, it is mandatory to point out that the concentrations of phytanic acid, pristanic acid, C22:0, C24:0, and C26:0 in cerebrospinal fluid of patients with peroxisomal disorders are below the detection limit or do not exceed the values of $16,34,100,350$, and $300 \mathrm{nM}$, respectively (for comparison, with exception of the C26:0 levels in patients with an acyl-CoA oxidase deficiency, these concentrations are less than one percent of those found in the serum of these patients) [50, 53].

In the following sections, we focus on what is currently known about the peroxisome-oxidative stress-neurodegeneration nexus in patients suffering from a Zellweger spectrum disorder (see 4.1) or $\mathrm{X}$-linked adrenoleukodystrophy (see 4.2). When relevant, animal studies are also discussed.

\subsection{Zellweger Spectrum Disorders}

The Zellweger spectrum disorders (ZSDs) are a group of rare (combined incidence: 1:50,000 live births), clinically and genetically heterogeneous disorders caused by mutations in PEX genes. The encoded proteins, referred to as peroxins, are involved in peroxisome biogenesis and maintenance. Mutations leading to a loss of peroxin function result in the absence of functional peroxisomes and, consequently, the accumulation of peroxisomal substrates (e.g., VLCFAs, phytanic- and pristanic acid, and C27-bile acid intermediates) and loss of peroxisomal products (e.g., plasmalogens and DHA) [54]. ZSD patients present with a continuum of symptoms ranging from sensorineural hearing loss, vision impairment, and low muscle tone to profound liver, kidney, and brain dysfunction [54].

The molecular mechanisms underlying the neuropathology of ZSD are not yet well understood. Solid evidence that the absence of functional peroxisomes in ZSD patients causes lipid-induced mito- 
chondrial dysfunction and oxidative damage in the CNS is lacking. However, by employing mice with a brain-specific deletion of PEX13, Crane and co-workers could show that the neuronal cell death (and subsequent impairment of cerebellar development) caused by absence of functional peroxisomes is associated with aberrant mitochondrial ROS production and dynamics [37]. These findings are in line with the observations that deletion of even a single allele of PEX11 $\beta$ is sufficient to cause oxidative stress and neuronal death in mouse brain [1]. In contrast, no specific relationship could be observed between peroxisome dysfunction, mitochondrial failure, generalized oxidative stress, and CNS lesions in mice with a brain-restricted deletion of PEX5: mitochondria in cortical plate neurons did not exhibit obvious abnormalities; oxidative stress markers were only upregulated in Purkinje cells; and treatment of the animals with an antioxidant cocktail did neither improve their motor performance nor have an effect on their health status [5]. As such, the present data are not sufficiently robust to accept or reject the hypothesis that a general defect in peroxisome biogenesis in ZSD patients causes neuronal cell death and brain dysfunction through mitochondria-mediated oxidative stress.

\subsection{X-linked Adrenoleukodystrophy}

X-linked adrenoleukodystrophy (X-ALD), the most prevalent peroxisomal disorder ( 1:17,000 live male births), is a clinically heterogeneous and progressive neurometabolic disease caused by mutations in the $A B C D 1$ gene [12]. This gene encodes a peroxisomal ATP-binding cassette-containing transmembrane protein (ALDP) that mediates the import of CoA-esters of VLCFAs into the organelle. Mutations in ALDP may result in the accumulation of VLCFAs in body tissues, including the brain and spinal cord (e.g., as cerebrosides, phosphatidylcholine, sphingomyelin, sulfatides, and cholesteryl esters) [33], due to impaired peroxisomal $\beta$-oxidation. Depending on yet-to-be-identified environmental factors and modifier genes, a reduction or absence of ALDP activity may induce among others - adrenocortical insufficiency, progressive inflammatory demyelination, axonal damage, and neurodegeneration [12]. 
The molecular mechanisms of how the VLCFA-containing lipids can drive these symptoms are currently under intense investigation. Besides the accumulation of VLCFAs, other early hallmarks of XALD are oxidative damage and mitochondrial dysfunction, both in patients as well as in experimental cell and mouse models [39]. Approximately a decade ago, Singh and Pujol proposed a "three-hit hypothesis" to explain the physiopathogenesis of cerebral ALD, the most severe X-ALD phenotype. This hypothesis postulates that, as a direct consequence of peroxisomal metabolic impairment, oxidative stress functions as the first hit, neuroinflammation acts as the second insult, and a generalized loss of peroxisome function serves as the third hit [46]. The observations that (i) the levels of 7ketocholesterol $(0.5-4.0 \mu \mathrm{M})$, a reliable biomarker of oxidative damage, are on average 10- to 20-fold higher in the plasma of X-ALD patients than in the plasma of healthy control subjects [35], (ii) this bioactive product of lipid oxidation, which can trigger inflammation and an oxiapoptophagic (OXIdation, APOPTOsis, and autoPHAGY) mode of cell death, also induces general peroxisome dysfunction [35], and (iii) upregulation of endogenous antioxidant responses can rescue mitochondrial function, prevent inflammatory imbalance, and halt axonal degeneration and locomotor deficits in an X-ALD mouse model, are in line with this hypothesis [39]. However, it remains to be investigated whether or not the inflammatory and cell death responses elicited by $12.5-50 \mu \mathrm{M}$ of 7-ketocholesterol in murine microglial BV-2 cells [35] also occur under conditions more closely mimicking the in vivo microenvironment and at 7-ketocholesterol concentrations $(05 .-4.0 \mu \mathrm{M})$ found in the plasma of X-ALD patients.

\section{Neurodegenerative Disease and Peroxisomal Redox Dysfunction}

Increasing evidence indicates that, among other processes, peroxisome function is declined with aging ([28]; and references therein). In addition, a number of studies have suggested that this phenomenon may contribute to the onset and progression of more widespread age-related neurological disorders ([2,23]; and references therein). Common brain abnormalities in individuals suffering from such dis- 
eases include lipid changes, mitochondrial dysfunction, oxidative stress, inflammation, deposits of misfolded protein aggregates, cell death, and demyelination (Fig. 3). Given that many of these manifestations can also be linked to peroxisome dysfunction (see 4), this organelle too may play a role in the etiology and pathogenesis of agerelated neurodegenerative diseases. Importantly, many of the abnormalities listed are not independent, but promote each other. This is perhaps best illustrated by the discoveries that oxidative stress can be either causative, or consecutive, to protein aggregation, and that both phenomena can be part of a vicious cycle ([27]; and references therein). In the following sections, we further elaborate on what is currently known about the peroxisome-oxidative stressneurodegeneration nexus in Alzheimer's disease (see 5.1), Parkinson's disease (see 5.2), and multiple sclerosis (see 5.3). Importantly, given the unavailability of sufficient empirical studies on peroxisome dysfunction in amyotrophic lateral sclerosis (ALS), this progressive motor neuron disorder will not be discussed. However, for completeness, it is necessary to mention that (i) a deficiency in Damino acid oxidase (DAO), a peroxisomal $\mathrm{H}_{2} \mathrm{O}_{2}$-producing enzyme, triggers motor neuron degeneration in both mice and familial ALS patients, and (ii) the spinal cord levels of D-serine are $\sim 5$-fold increased in $\mathrm{DAO}^{-/-}$mice relative to control strains [40].

\subsection{Alzheimer's Disease}

Alzheimer's disease (AD), the most common form of dementia (prevalence: $\sim 10 \%$ of all people aged 65 and over), is a progressive neurodegenerative disorder whose pathology is related to the deposition of intracellular hyperphosphorylated tau aggregates and extracellular amyloid- $\beta$ plaques [55]. Despite the fact that the molecular pathogenesis underlying this devastating disease is not yet fully understood, there is evidence that neuronal damage and death are associated with mitochondrial dysfunction, increased oxidative stress, and alterations in lipid metabolism [36, 55].

Currently, there is compelling evidence to support the notion that both peroxisome function and dysfunction can be linked to AD. For example, lipid analyses of human cortical brain samples have docu- 
mented that the levels of ethanolamine plasmalogens with a PUFA at the sn-2 position and the concentrations of $\mathrm{C} 24: 0$ plus $\mathrm{C} 26: 0$ are reduced $(\sim 7.7 \%)$ and elevated $(\sim 36.6 \%)$, respectively, in late-stage (V and VI) compared to early-stage (I and II) AD patients [25]; and another study reported that, in the prefrontal cortex of AD patients, choline plasmalogens (decrease: $\sim 73 \%$ ) are more affected than ethanolamine plasmalogens (decrease: 20\%) [20]. In addition, the first study demonstrated that the transport of peroxisomes to neurites is compromised in AD-affected neurons. Numerical and functional changes of peroxisomes were also found in the neocortex of the widely-used $\mathrm{Tg} 2576$ mouse model of AD ([36]; and references therein); and in rat, a decline in peroxisomal $\beta$-oxidation (as suggested by elevation of cortical C26:0 levels) positively correlated with significant elevations of the lipid peroxidation marker malondialdehyde, the accumulation of amyloid- $\beta$ peptides, and spatial memory deficits [44]. In line with these observations, others found that an increase in peroxisome number with concomitant increased catalase activity (and plasmalogen levels) mitigates amyloid- $\beta$-induced toxicity in primary rat hippocampal neuron cultures and in brains of transgenic APPswe/PS1dE9 mice, another commonly used mouse model of AD ([21]; and references therein). This phenomenon appears to be at least partially mediated through enhanced redox buffering capacity and mitochondrial fitness. Note that the oxidative stress link is further reinforced by the observation that intraperitoneal injections of CAT-SKL, a catalase variant with enhanced peroxisomal targeting capacity, attenuate the loss of cholinergic neurons and long-term reference memory deficits in rats that received intracerebroventricular injections of amyloid- $\beta$ [34].

\subsection{Parkinson's Disease}

Parkinson's disease (PD), the second-most widespread neurodegenerative disorder after AD (prevalence: $~ 1-2 \%$ of all persons over 60 years of age), is characterized by a progressive loss of dopamine-producing neurons in the substantia nigra pars compacta, a process causing motor deficits such as resting tremor, rigidity, and bradykinesia ([4]; and references therein). A typical pathological 
hallmark of PD is the presence of intraneural $\alpha$-synuclein aggregates. The exact molecular mechanisms in the pathology of PD remain to be clarified. Once again, growing evidence supports the view that mitochondrial dysfunction and oxidative stress play a pivotal role in PD pathogenesis [4].

To the best of our knowledge, there are only a handful of studies examining the potential links between $\alpha$-synuclein-related brain pathology and peroxisome dysfunction. On one hand, it has been reported that in PD patients frontal cortex lipid rafts contain less plasmalogens and DHA than those in the control group [30]. On the other hand, it has been observed that $\alpha$-synuclein accumulates in neurons and glial cells from X-ALD patients [48], and that generalized defects in peroxisome biogenesis promote the oligomerization and phosphorylation of $\alpha$-synuclein in mouse brain, a process that appears to be positively correlated with an increase in VLCFAs and long-chain n-6-PUFAs, and a drop in most n-3-PUFAs [58]. However, in a follow-up study, the same authors concluded that high or prolonged dietary levels of DHA enhance the $\alpha$-synuclein pathology in A53T $\alpha$-synuclein transgenic mice [57]. In addition, they demonstrated that, in the brain of these mice, peroxisomal $\alpha$ - and $\beta$ oxidation are normal, plasmalogen levels are slightly increased, and catalase activity is decreased by $25-30 \%$ [56].

Importantly, some studies have hinted that compounds known as peroxisome proliferating agents (PPAs) may provide neuroprotection, at least in PD animal models ([38]; and references therein). The precise underlying mechanisms are unknown, but are most likely multifactorial. Indeed, the effects of PPAs such as fibrates and thiazolidinediones are mediated by distinct peroxisome proliferator activated receptors (PPARs), which tune the gene expression of proteins involved in a wide range of cellular processes, including - among others - peroxisome biogenesis, mitochondrial biogenesis, lipid metabolism, and antioxidant and inflammatory responses. In this context, it is interesting to note that, in peroxisome-defective fibroblasts overexpressing $\alpha$-synuclein, the pathogenic accumulation of intracellular $\alpha$-synuclein inclusions did not correlate with oxidative stress or mitochondrial dysfunction [58]. However, whether or not this is also true for nigral dopaminergic neurons, remains to be determined. Finally, it has been demonstrated that PPI-1011, a DHA-containing 
ethanolamine plasmalogen precursor, protects against striatal dopamine loss in a 1-methyl-4-phenyl-1,2,3,6-tetrahydropyridineintoxicated mouse model of PD [31].

\subsection{Multiple Sclerosis}

Multiple sclerosis (MS), whose prevalence can vary considerably (e.g., from 2 to $>200$ per 100,000 residents) between different regions and populations, is a chronic inflammatory condition of the CNS with a varying clinical picture ([15]; and references therein). Pathological hallmarks of the disease include - among others - impaired homeostasis of glial cells with production of ROS, mitochondrial damage, demyelination, and axonal degeneration [15].

Once again, the precise molecular mechanisms leading to the disease pathology of MS are not yet well understood. However, as for $\mathrm{AD}$ and $\mathrm{PD}, \mathrm{MS}$ has been associated with impaired peroxisomal function, both in patient-derived tissues [18] and in the widelyaccepted experimental allergic encephalomyelitis (EAE) animal model for MS [45]. Indeed, in the brain of EAE rats, C26:0 levels are increased (by $\sim 40 \%$ ), and plasmalogen levels and catalase activity are decreased (by 16-30\% and 37\%, respectively) [45]; and in grey matter from brain autopsies of MS patients, C26:0 levels are 2-fold elevated and peroxisomal ABCD3 membrane proteinpositive neurons are negatively correlated to disease duration [18]. In addition, more recently, it has been demonstrated that a combined administration of lovastatin and the AMP-activated protein kinase activator AICAR to EAE mice (i) increases peroxisomal and mitochondrial biogenesis and function through PPAR $\alpha / \gamma$ - and PPAR $\gamma$ coactivator-1 alpha-mediated pathways, (ii) alleviates the inflammation-induced mitochondrial and peroxisomal defects, and (iii) provides protection against myelin and axonal pathology in EAE [47]. In the cerebrospinal fluid of MS patients, only PPAR $\gamma$ appears to be upregulated [49]. Based on these findings, it is tempting to speculate that alterations in peroxisome function may contribute to neuronal dysfunction and degeneration in MS, and that restoring peroxisome function may be beneficial for MS disease prognosis [47]. However, given that targeted lipidomic profiling studies of cerebrospinal fluid 
samples from MS patients do not provide any evidence for peroxisomal dysfunction [17], this view should be considered with caution.

\section{Conclusions and Outlook}

The studies reviewed in this chapter clearly demonstrate that peroxisomes play an important role in normal brain function (Fig. 1). In addition, they strengthen the view that peroxisome function is compromised in the brain of patients suffering from common agerelated neurodegenerative diseases (Fig. 3). The specific mechanisms underlying peroxisome dysfunction-related brain impairment remain obscure, but potential elements include the shortage of peroxisomal products (e.g., plasmalogens), the toxic accumulation of peroxisomal substrates (e.g., VLCFA-containing lipids), and perturbations in peroxisomal $\mathrm{H}_{2} \mathrm{O}_{2}$ metabolism and signaling (Fig. 2). Importantly, imbalances in any of these factors can be firmly linked to inflammation [46], mitochondrial dysfunction [43], and protein aggregation [27], all hallmarks of neurodegeneration [8]. Given that virtually all these processes are tightly interconnected, it is difficult to pinpoint the primary triggering event(s).

A major challenge is to decipher which shortage or excess of peroxisome-related metabolites and signaling molecules drives CNS pathology. In this context, it is mandatory to note that most of the in vitro lipotoxicity studies have been performed with fatty acid concentrations in the low micromolar range, which closely reflect the situation in plasma $[50,53]$. As already pointed out above (see 4), these concentrations are often 100-1000-fold higher than those found in brain $[50,53]$, thereby raising doubts regarding the physiological relevance of these studies. In addition, given that the concentrations of phytanic and pristanic acid in cerebrospinal fluid from patients with a peroxisomal disorder only seldom exceed the control range, it is unlikely that these substances play a direct pathogenic role in the neurological manifestations of such patients [50].

With respect to the age-related neurodegenerative disorders, it is unclear to which extent the observed decline in peroxisome function actively contributes to the disease phenotype or merely represents a secondary change associated with the pathologic process. However, 
in this context, it is relevant to mention that various inflammatory stimuli have been reported to reduce plasmalogen levels in glial cell lines and microglia in mouse cortex through NF- $\kappa \mathrm{B}$ activation and subsequent c-myc-mediated transcriptional repression of GNPAT, a gene coding for a peroxisomal membrane protein essential for etherphospholipid biosynthesis [19]. In addition, it has been demonstrated that peroxisome number and function are also altered at sites of secondary neurodegeneration (e.g., post-stroke or post-doxorubicin chemotherapy). Indeed, induction of transient focal cerebral ischemia in mice significantly increases peroxisome number as well as catalase activity in peri-infarct neurons [59]; and treatment of mice with the antineoplastic agent doxorubicin increases the number of peroxisomes in neurons [32]. Interestingly, in the first case, the observed changes were considered to be an adaptive response, protecting neurons against ischemia/reperfusion-induced oxidative and metabolic stress [59]. In contrast, in the second case, the expansion of the peroxisomal compartment was attributed to a downregulation of pexophagy, a phenomenon that coincided with enhanced peroxisome-derived oxidative stress and was considered to contribute to the deterioration of cognitive function and accelerated brain aging in cancer patients and survivors [32].

A final key message is that peroxisomal fitness is pivotal to protect the CNS from inflammation, mitochondrial dysfunction, oxidative stress, and neurodegeneration. This implies that diet strategies [10] and therapeutic approaches [21, 38, 47] aimed at preserving, normalizing, or improving peroxisome abundance and activity may have beneficial effects for patients suffering from neurodegenerative conditions.

\section{Acknowledgements}

This work was supported by grants from the KU Leuven (C14/18/088), the Fonds voor Wetenschappelijk OnderzoekVlaanderen (Onderzoeksprojecten G095315N, G091819N, and GOA8619N), the ERA-Net for Research Programmes on Rare Diseases (PERescue), and H2020-MSCA-ITN (812968). CL is support- 
ed by a postdoctoral fellowship of the Research Foundation - Flanders $(1213620 \mathrm{~N})$.

\section{References $^{1}$}

[1] Ahlemeyer B, Gottwald M, Baumgart-Vogt E (2012) Deletion of a single allele of the Pex $11 \beta$ gene is sufficient to cause oxidative stress, delayed differentiation and neuronal death in mouse brain. Dis Model Mech 5:125-140.

[2] Berger J, Dorninger F, Forss-Petter S et al. (2016) Peroxisomes in brain development and function. Biochim Biophys Acta 1863:934-955.

[3] Borges CG, Canani CR, Fernandes CG et al. (2015) Reactive nitrogen species mediate oxidative stress and astrogliosis provoked by in vivo administration of phytanic acid in cerebellum of adolescent rats: a potential contributing pathomechanism of cerebellar injury in peroxisomal disorders. Neuroscience 304: 122-132.

[4] Bose A, Beal MF (2019) Mitochondrial dysfunction and oxidative stress in induced pluripotent stem cell models of Parkinson's disease. Eur J Neurosci 49:525-532.

[5] Bottelbergs A, Verheijden S, Van Veldhoven PP et al. (2012) Peroxisome deficiency but not the defect in ether lipid synthesis causes activation of the innate immune system and axonal loss in the central nervous system. J Neuroinflammation 9:61.

[6] Brodde A, Teigler A, Brugger B et al. (2012) Impaired neurotransmission in ether lipid-deficient nerve terminals. Hum Mol Genet 21:2713-2724.

[7] Busanello EN, Lobato VG, Zanatta Â et al. (2014) Pristanic acid provokes lipid, protein, and DNA oxidative damage and reduces the antioxidant defenses in cerebellum of young rats. Cerebellum 13:751-759.

\footnotetext{
${ }^{1}$ We apologize to colleagues whose work could not be cited due to space limitations.
} 
[8] Cenini G, Lloret A, Cascella R (2019) Oxidative stress in neurodegenerative diseases: from a mitochondrial point of view. Oxid Med Cell Longev 2019:2105607.

[9] Cobley JN, Fiorello ML, Bailey DM (2018) 13 reasons why the brain is susceptible to oxidative stress. Redox Biol 15:490-503.

[10] Debbabi M, Nury T, Zarrouk A et al. (2016) Protective effects of $\alpha$-tocopherol, $\gamma$-tocopherol and oleic acid, three compounds of olive oils, and no effect of Trolox, on 7ketocholesterol-induced mitochondrial and peroxisomal dysfunction in microglial BV-2 cells. Int J Mol Sci 17:E1973.

[11] De Munter S, Verheijden S, Régal L et al. (2015) Peroxisomal disorders: a review on cerebellar pathologies. Brain Pathol 25:663-678.

[12] Deon M, Marchetti DP, Donida B et al. (2016) Oxidative stress in patients with X-linked adrenoleukodystrophy. Cell Mol Neurobiol 36:497-512.

[13] Di Cara F, Andreoletti P, Trompier D et al. (2019) Peroxisomes in immune response and inflammation. Int J Mol Sci 20:E3877.

[14] Dorninger F, Forss-Petter S, Berger J (2017) From peroxisomal disorders to common neurodegenerative diseases - the role of ether phospholipids in the nervous system. FEBS Lett 591:2761-2788.

[15] Faissner S, Plemel JR, Gold R et al. (2019) Progressive multiple sclerosis: from pathophysiology to therapeutic strategies. Nat Rev Drug Discov (in press; doi: 10.1038/s41573019-0035-2).

[16] Fransen M, Lismont C, Walton P (2017) The peroxisomemitochondria connection: how and why? Int J Mol Sci 18:E1126.

[17] Gonzalo H, Brieva L, Tatzber F et al. (2012) Lipidome analysis in multiple sclerosis reveals protein lipoxidative damage as a potential pathogenic mechanism. J Neurochem 123:622634.

[18] Gray E, Rice C, Hares K et al. (2014) Reductions in neuronal peroxisomes in multiple sclerosis grey matter. Mult Scler 20:651-659. 
[19] Hossain MS, Abe Y, Ali F et al. (2017) Reduction of ethertype glycerophospholipids, plasmalogens, by NF- $\kappa$ B signal leading to microglial activation. J Neurosci 37:4074-4092.

[20] Igarashi M, Ma K, Gao F et al. (2011) Disturbed choline plasmalogen and phospholipid fatty acid concentrations in Alzheimer's disease prefrontal cortex. J Alzheimers Dis 24:507-517.

[21] Inestrosa NC, Carvajal FJ, Zolezzi JM et al. (2013) Peroxisome proliferators reduce spatial memory impairment, synaptic failure, and neurodegeneration in brains of a double transgenic mice model of Alzheimer's disease. J Alzheimers Dis 33:941-959.

[22] Islinger M, Voelkl A, Fahimi HD et al. (2018) The peroxisome: an update on mysteries 2.0. Histochem Cell Biol 150:443-471.

[23] Jo DS, Cho DH (2019) Peroxisomal dysfunction in neurodegenerative diseases. Arch Pharm Res 42:393-406.

[24] Kassmann CM, Quintes S, Rietdorf J et al. (2011) A role for myelin-associated peroxisomes in maintaining paranodal loops and axonal integrity. FEBS Lett 585:2205-2211.

[25] Kou J, Kovacs GG, Höftberger R et al. (2011) Peroxisomal alterations in Alzheimer's disease. Acta Neuropathol 122: 271-283.

[26] Leipnitz G, Amaral AU, Fernandes CG et al. (2011) Pristanic acid promotes oxidative stress in brain cortex of young rats: a possible pathophysiological mechanism for brain damage in peroxisomal disorders. Brain Res 1382:259-265.

[27] Lévy E, El Banna N, Baïlle D, et al. (2019) Causative links between protein aggregation and oxidative stress: a review. Int J Mol Sci 20: E3896.

[28] Lismont C, Nordgren M, Van Veldhoven PP et al. (2015) Redox interplay between mitochondria and peroxisomes. Front Cell Dev Biol 3:35.

[29] Lismont C, Revenco I, Fransen M (2019) Peroxisomal hydrogen peroxide metabolism and signaling in health and disease. Int J Mol Sci 20:E3673.

[30] Marin R, Fabelo N, Martín V et al. (2017) Anomalies occurring in lipid profiles and protein distribution in frontal cortex 
lipid rafts in dementia with Lewy bodies disclose neurochemical traits partially shared by Alzheimer's and Parkinson's diseases. Neurobiol Aging 49:52-59.

[31] Miville-Godbout E, Bourque M, Morissette M et al. (2016) Plasmalogen augmentation reverses striatal dopamine loss in MPTP mice. PLoS One 11:e0151020.

[32] Moruno-Manchon JF, Uzor NE, Kesler SR et al. (2018) Peroxisomes contribute to oxidative stress in neurons during doxorubicin-based chemotherapy. Mol Cell Neurosci 86:6571.

[33] Moser HW, Mahmood A, Raymond GV (2007) X-linked adrenoleukodystrophy. Nat Clin Pract Neurol 3:140-151.

[34] Nell HJ, Au JL, Giordano CR et al. (2016) Targeted antioxidant, catalase-SKL, reduces beta-amyloid toxicity in the rat brain. Brain Pathol 27:86-94.

[35] Nury T, Zarrouk A, Ragot K et al. (2017) 7-Ketocholesterol is increased in the plasma of X-ALD patients and induces peroxisomal modifications in microglial cells: Potential roles of 7-ketocholesterol in the pathophysiology of X-ALD. J Steroid Biochem Mol Biol 169:123-136.

[36] Porcellotti S, Fanelli F, Fracassi A et al. (2015) Oxidative stress during the progression of $\beta$-amyloid pathology in the neocortex of the Tg2576 mouse model of Alzheimer's disease. Oxid Med Cell Longev 2015:967203.

[37] Rahim RS, Chen M, Nourse CC et al. (2016) Mitochondrial changes and oxidative stress in a mouse model of Zellweger syndrome neuropathogenesis. Neuroscience 334:201-213.

[38] Rane P, Sarmah D, Bhute S et al. (2019) Novel targets for Parkinson's disease: addressing different therapeutic paradigms and conundrums. ACS Chem Neurosci 10:44-57.

[39] Ranea-Robles P, Launay N, Ruiz M et al. (2018) Aberrant regulation of the GSK-3 $\beta / \mathrm{NRF} 2$ axis unveils a novel therapy for adrenoleukodystrophy. EMBO Mol Med 10:e8604.

[40] Sasabe J, Miyoshi Y, Suzuki M et al. (2012) D-amino acid oxidase controls motoneuron degeneration through D-serine. Proc Natl Acad Sci USA 109:627-632. 
[41] Schlüter A, Real-Chicharro A, Gabaldón T et al. (2010) PeroxisomeDB 2.0: an integrative view of the global peroxisomal metabolome. Nucleic Acids Res 38:D800-D805.

[42] Schönenberger MJ, Kovacs WJ (2017) Isolation of peroxisomes from mouse brain using a continuous Nycodenz gradient: a comparison to the isolation of liver and kidney peroxisomes. Methods Mol Biol 1595:13-26.

[43] Schönfeld P, Reiser G (2016) Brain lipotoxicity of phytanic acid and very long-chain fatty acids. Harmful cellular/mitochondrial activities in Refsum disease and X-linked adrenoleukodystrophy. Aging Dis 7:136-149.

[44] Shi Y, Sun X, Sun Y et al. (2016) Elevation of cortical C26:0 due to the decline of peroxisomal $\beta$-oxidation potentiates amyloid $\beta$ generation and spatial memory deficits via oxidative stress in diabetic rats. Neuroscience 315:125-135.

[45] Singh I, Paintlia AS, Khan M et al. (2004) Impaired peroxisomal function in the central nervous system with inflammatory disease of experimental autoimmune encephalomyelitis animals and protection by lovastatin treatment. Brain Res 1022:1-11.

[46] Singh I, Pujol A (2010) Pathomechanisms underlying Xadrenoleukodystrophy: a three-hit hypothesis. Brain Pathol 20:838-844.

[47] Singh I, Samuvel DJ, Choi S et al. (2018) Combination therapy of lovastatin and AMP-activated protein kinase activator improves mitochondrial and peroxisomal functions and clinical disease in experimental autoimmune encephalomyelitis model. Immunology 154:434-451.

[48] Suzuki K, Iseki E, Togo T et al. (2007) Neuronal and glial accumulation of alpha- and beta-synucleins in human lipidoses. Acta Neuropathol 114:481-489.

[49] Szalardy L, Zadori D, Bencsik K et al. (2017) Unlike PPARgamma, neither other PPARs nor PGC-1alpha is elevated in the cerebrospinal fluid of patients with multiple sclerosis. Neurosci Lett 651:128-133.

[50] ten Brink HJ, van den Heuvel CM, Poll-The BT et al. (1993) Peroxisomal disorders: concentrations of metabolites in cer- 
ebrospinal fluid compared with plasma. J. Inherit Metab Dis 16, 587-590.

[51] Van Veldhoven PP (2010) Biochemistry and genetics of inherited disorders of peroxisomal fatty acid metabolism. J Lipid Res 51:2863-2895.

[52] Van Veldhoven PP, Baes M (2013) Peroxisome-deficient invertebrate and vertebrate animal models. Front Physiol 4:335.

[53] Verhoeven NM, Kulik W, van den Heuvel CM, et al. (1995) Pre- and postnatal diagnosis of peroxisomal disorders using stable-isotope dilution gas chromatography--mass spectrometry. J Inherit Metab Dis 18 (Suppl 1):45-60.

[54] Wanders RJA (2018) Peroxisomal disorders: improved laboratory diagnosis, new defects and the complicated route to treatment. Mol Cell Probes 40:60-69.

[55] Wojsiat J, Zoltowska KM, Laskowska-Kaszub K et al. (2018) Oxidant/antioxidant imbalance in Alzheimer's disease: therapeutic and diagnostic prospects. Oxid Med Cell Longev 2018:6435861.

[56] Yakunin E, Kisos H, Kulik W et al. (2014) The regulation of catalase activity by PPAR $\gamma$ is affected by $\alpha$-synuclein. Ann Clin Transl Neurol 1:145-159.

[57] Yakunin E, Loeb V, Kisos H et al. (2012) A-synuclein neuropathology is controlled by nuclear hormone receptors and enhanced by docosahexaenoic acid in a mouse model for Parkinson's disease. Brain Pathol 22: 280-294.

[58] Yakunin E, Moser A, Loeb V et al. (2010) Alpha-synuclein abnormalities in mouse models of peroxisome biogenesis disorders. J Neurosci Res 88:866-876.

[59] Young JM, Nelson JW, Cheng J et al. (2015) Peroxisomal biogenesis in ischemic brain. Antioxid Redox Signal 22:109120.

[60] Zarrouk A, Vejux A, Nury T et al. (2012) Induction of mitochondrial changes associated with oxidative stress on very long chain fatty acids (C22:0, C24:0, or C26:0)-treated human neuronal cells (SK-NB-E). Oxid Med Cell Longev 2012:623257. 


\section{Legends to figures}

Fig. 1 Emerging roles of peroxisomes in brain physiology. Schematic overview depicting the role of healthy peroxisomes (indicated in green) in the maintenance of normal brain physiology. DHA, docosahexaenoic acid; HPFAs, hydroperoxy fatty acids; VLCFAs, very-long-chain fatty acids; PA, phytanic acid; PO, peroxisome; PrA, pristanic acid.

Fig. 2 Emerging roles of primary peroxisome dysfunction in brain pathology. Schematic overview depicting how primary peroxisomal dysfunction (indicated in red) may contribute to brain pathology. DHA, docosahexaenoic acid; HPFAs, hydroperoxy fatty acids; VLCFAs, very-long-chain fatty acids; PA, phytanic acid; PO, peroxisome; PrA, pristanic acid.

Fig. 3 Neurodegenerative disease and secondary peroxisomal dysfunction. Schematic overview depicting the potential relationships between hallmarks of neurodegenerative diseases and secondary peroxisome dysfunction (indicated in orange). The red arrows show the potential vicious cycle between neurodegeneration and peroxisome impairment. The green T-bar provides literature-based suggestions of how this cycle may be broken. CAT, catalase; PO, peroxisome; VLCFA, very-long-chain fatty acid. 
Figure 1

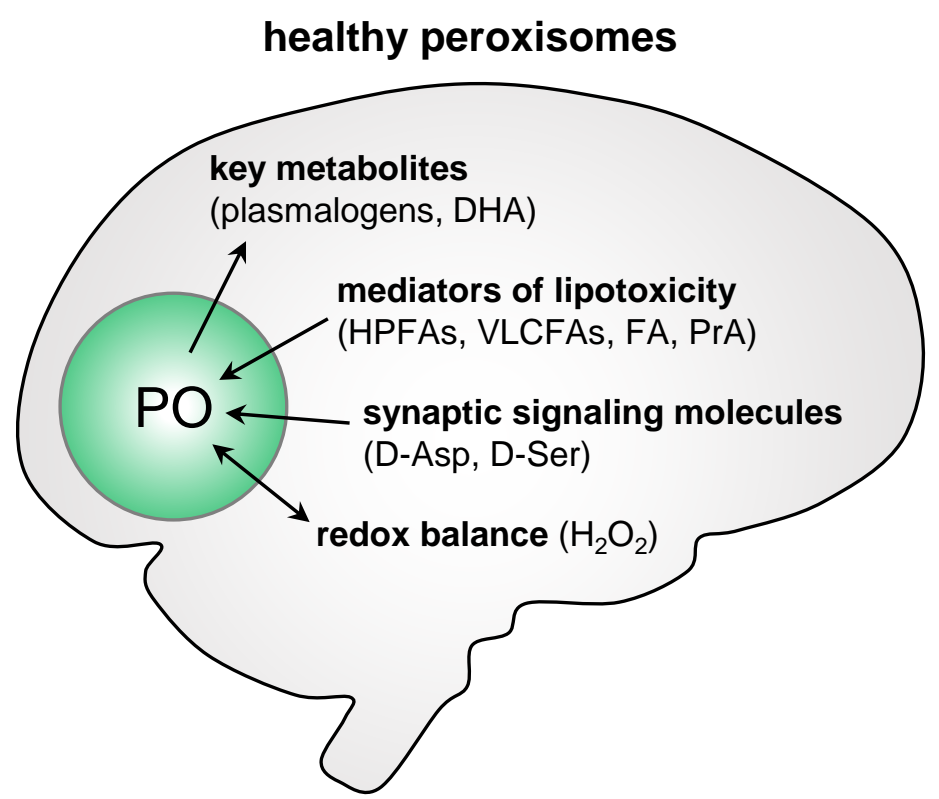




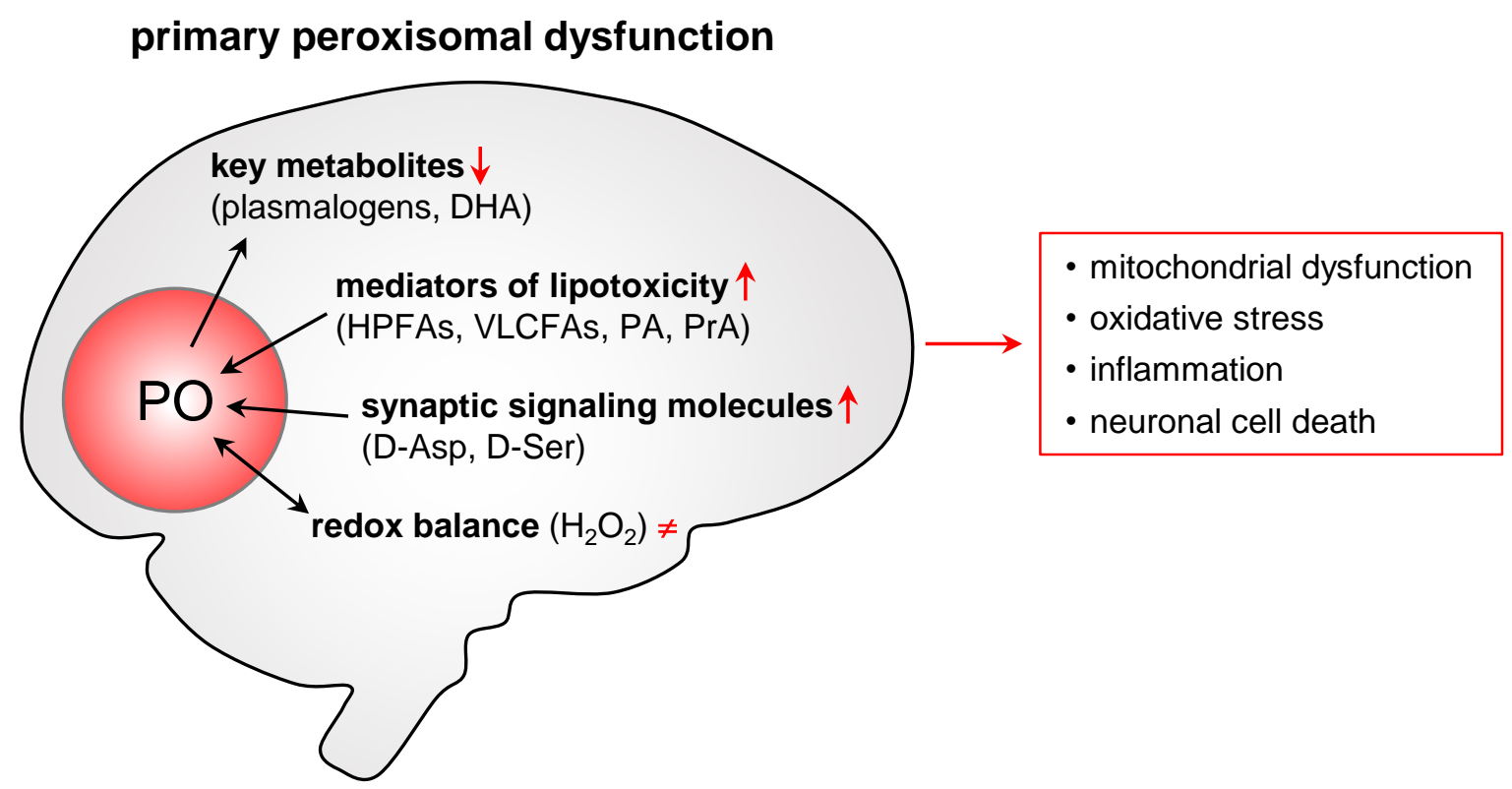




\section{neurodegenerative disease}

\section{secondary peroxisomal dysfunction}

- lipid changes

- mitochondrial dysfunction

- oxidative stress

- protein aggregation and mislocalization

- inflammation

- neuronal cell death

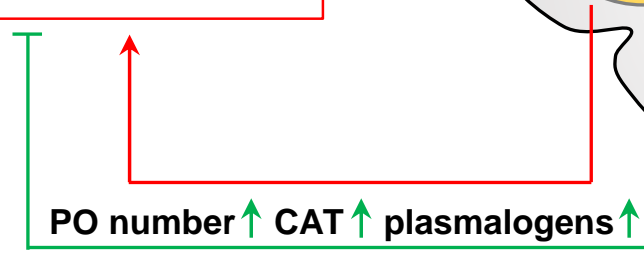

key metabolites (plasmalogens)

number $\uparrow$ CAT $\uparrow$ plasmalogens 